\title{
Deriving the distributivity potential of adjectives via measurement theory
}

\author{
Lelia Glass*
}

\begin{abstract}
The boxes are heavy can convey that each box is heavy (distributive), or that some individually light boxes qualify as heavy when lifted together (nondistributive; Schwarzschild 1996, Schwarzschild 2011). In contrast, the boxes are fragile generally requires each box to be fragile (distributive). Which adjectives behave like heavy or like fragile, and why? This paper proposes a measurementtheoretic account. For a gradable adjective to be understood nondistributively, I argue that $a \oplus b$ must exceed $a$ and $b$ along the scale associated with the adjective. That way, the contextual standard $\theta$ for what 'counts as' (adjective) in the context can be set in such a way that the composite object $a \oplus b$ surpasses the contextual standard $\theta$ while $a$ and $b$ individually fall short of it - a nondistributive understanding, in that the adjective is true of $a \oplus b$ together but not of $a$ or $b$ individually. This ordering is possible for heavy but not fragile, deriving their differences. More generally, researchers agree that an adjective's potential for distributivity depends on what we know about the property it describes. Making that idea more explanatory, this paper articulates which features of the property described by the adjective matter for distributivity and why.
\end{abstract}

Keywords. distributive, collective, stubborn distributivity, gradable adjectives, measurement theory, lexical semantics

1.Introduction. When a sentence has a plural subject such as the boxes or Box $A$ and Box B, we draw inferences about how the predicate of the sentence applies to each member of the subject (e.g., Scha 1981, Link 1983). Focusing on adjectives, some are understood DISTRIBUTIVELY (1), meaning that the adjective is inferred to be true of - to 'distribute' down to - each individual member of the subject.

(1) Box A and Box B are fragile.

(distributive)

a. $\sqrt{ }$ Distributive: Box $A$ is fragile, Box $B$ is fragile.

b. XNondistributive: Box A and Box B are jointly fragile but not individually so.

More adjectives that behave like fragile include light, short, small, full, empty, clean, dirty, old, and new: applied to two boxes, these adjectives are also generally inferred to be true of each box.

In contrast, other adjectives are understood NONDISTRIBUTIVELY (2), meaning that they are not inferred to be individually true of each member of the subject (at least, not unless we imagine some sort of implicit object), but instead only apply to the subject (the two boxes) as a whole.

Box A and Box B are connected.

a. XDistributive: Box A is connected, Box B is connected.

(nondistributive)

\footnotetext{
* I am grateful to Beth Levin, Christopher Potts, Cleo Condoravdi, and Daniel Lassiter for advice, and to the audience at the LSA Annual Meeting (Salt Lake City, 2018) for feedback. Thanks to the American Council of Learned Societies (ACLS) for financial support. Author: Lelia Glass, Stanford University (lelia@stanford.edu).
} 
b. $\sqrt{ }$ Nondistributive: Box $\mathrm{A}$ and Box $\mathrm{B}$ are jointly connected but not individually so.

The class of connected-type adjectives is not discussed further in this paper, since these involve a sense of reciprocity not shared by the others.

Still other adjectives can be understood in both ways. If two boxes are said to be heavy (3), perhaps each box counts as heavy (distributive); or perhaps they are light (or mid-weight) individually while qualifying as heavy when lifted together.

Box A and Box B are heavy.

(can be understood both ways)

a. VDistributive: Box A is heavy, Box B is heavy.

b. $\checkmark$ Nondistributive: Box A and Box B are jointly heavy but not individually so.

Further adjectives that behave like heavy include expensive (two boxes could each be expensive; or two individually affordable boxes could qualify as expensive when purchased together), beautiful (two boxes might each be beautiful, or two individually plain boxes might complement one another aesthetically), and $u g l y$ (two boxes might each be $u g l y$, or two individually nicelooking boxes might clash with one another, becoming ugly in combination).

Adding one more type of adjectives to create a four-way typology, there are also adjectives which could imaginably be understood nondistributively, but which in reality strongly favor a distributive understanding. Just as two light boxes might become heavy when lifted together, one can imagine that two short (or mid-height) boxes might become tall when stacked on top of one another. But in reality, it is difficult to understand tall in that way. Instead, tall is most easily understood distributively - true of each box individually. Schwarzschild (2011) names such predicates 'stubbornly distributive' on the grounds that they 'stubbornly' refuse to be understood nondistributively, even though we can imagine what it would mean for them to be understood that way.

Box $\mathrm{A}$ and Box B are tall.

('stubbornly distributive')

a. VDistributive: Box $A$ is tall, Box B is tall.

b. (??) Nondistributive: Box A and Box B are jointly tall but not individually so.

Other 'stubbornly distributive' adjectives include big, large, long, and wide.

There is a long tradition of work on how distributive and nondistributive understandings of sentences should be represented semantically (Scha 1981, Link 1983, Roberts 1987, Landman 1989, Lasersohn 1995, Schwarzschild 1996, Winter 2000, Landman 2000, Champollion 2010, de Vries 2015, Champollion 2017). Much of this work has focused on Verb Phrases rather than adjectives, although the same ideas apply to both. In this paper, I take on a different question, which is so far left open: Which adjectives are understood in which way(s), and why? Again leaving out the connected type, which other adjectives behave like fragile, like heavy, or like tall?

Researchers agree that inferences about distributivity are grounded in 'world knowledge' about the property described by an adjective (Scha 1981, Dowty 1987, Roberts 1987, Winter 2000); for example, fragile describes a characteristic of physical material; boxes each have their own physical material; so fragile is understood to be true of individual boxes (distributive). But it remains an open question what world knowledge matters and why, and how one might predict the distributivity potential of a new adjective. 
Making progress in this realm, Scontras and Goodman (2017) offer a convincing pragmatic explanation for why heavy (3) differs from tall (4): that the nondistributive ('collective') understanding of tall is too difficult for speakers and hearers to coordinate on pragmatically, given that the joint height of boxes depends on their transitory spatial arrangement (for example, it depends on whether the boxes are stacked on top of each other or sitting side by side); whereas the joint weight of boxes is stable regardless of how they are arranged. They show experimentally that in a context where boxes are reliably arranged in a stack, the nondistributive understanding of tall becomes more pragmatically accessible, consistent with their analysis ${ }^{1}$.

Even assuming that Scontras and Goodman are correct about why heavy differs from tall, however, we still face a more basic open question: What separates the adjectives like fragilefor which we cannot even imagine a nondistributive understanding - from those like tall and heavy, for which we can (whether or not it is pragmatically available)? Looking at Table 1, what separates the adjectives above the bolded line (the ones for which we can only imagine a distributive understanding) from those below the line (for which we can imagine both a distributive understanding and a nondistributive one, whether or not the nondistributive understanding is pragmatically available)?

\begin{tabular}{|c|c|}
\hline Distributive & Box $A$ and Box B are fragile (light, short, full, empty, old, new). \\
\hline & VDist.: each fragile $\quad$ XNondist.: jointly fragile \\
\hline \multirow[t]{2}{*}{ Both ways } & Box $A$ and Box B are heavy (expensive, beautiful, ugly). \\
\hline & $\checkmark$ Dist.: each heavy $\quad \sqrt{ }$ Nondist.: jointly heavy \\
\hline \multirow{2}{*}{$\begin{array}{l}\text { 'Stubbornly } \\
\text { distributive' }\end{array}$} & Box $A$ and Box B are tall (big, large, long, wide). \\
\hline & $\checkmark$ Dist.: each tall \\
\hline
\end{tabular}

Table 1: Distributive and nondistributive understandings available to different adjectives

On the one hand, there is evidence that the distributivity potential of adjectives is systematically related to the nature of the properties they describe. Just as we can imagine a nondistributive understanding (pragmatically available or not) for heavy and tall, the same goes for other adjectives that also describe physical dimensions in an increasing direction (large, big, wide, long). The fact that semantically similar adjectives pattern together suggests that their behavior is somehow tied to their meaning.

On the other hand, the distributivity potential of some adjectives appears idiosyncratic. Many adjectives come in antonym pairs (Clark 1972, Cruse 1976, Cresswell 1976, Seuren 1978, Lehrer and Lehrer 1982, Muehleisen 1997) such as heavy/light, open/closed, and tall/short, describing inversely related properties. Some antonym pairs pattern together in their

\footnotetext{
${ }^{1}$ While it is possible for tall to be understood nondistributively with enough context (e.g., in a situation where boxes regularly come out of a machine in a stack), there are other 'stubbornly distributive' adjectives where the imaginable nondistributive understanding is much more elusive. For example, even with a context favoring a nondistributive understanding, Syrett (2015) finds experimentally the boxes are round is robustly rejected to describe square boxes arranged into a round circle (presumably for the reason that Scontras and Goodman propose: the joint shape of boxes depends on their transitory spatial arrangement while their individual shape does not). But Scontras and Goodman's analysis is still consistent with the finding that tall is more pragmatically pliant than round. They do not predict that every 'stubbornly distributive' adjective will become nondistributive with enough context, as tall does; they just predict that nondistributive understandings are more available for adjectives describing properties of groups that are stable with respect to arrangement.
} 
distributivity potential: open and closed are both only understood distributively, in that if multiple boxes are open or closed, they each are. Beautiful and ugly can both be understood nondistributively as well as distributively: multiple boxes might each be beautiful or ugly (distributive), or might only be so when arranged together (nondistributive). Presumably the two halves of these antonym pairs pattern together because of the nature of the properties they describe: separate containers can only be open or closed individually; aesthetic judgments can be made about individual objects or collections thereof.

But then it is surprising that there are also antonym pairs which diverge in their potential for distributivity. For example, while heavy can be understood both distributively and nondistributively (3), it is quite difficult to imagine how its antonym light could be true of multiple boxes without also being true of each one ${ }^{2}$. If two boxes are light, then - because of the way weight works - they generally each are (see Maldonado et al, 2018).

(5) Box A and Box B are light.

(distributive)

a. VDistributive: Box A is light, Box B is light.

b. XNondistributive: Box A and Box B are jointly light but not individually so.

Within this apparent idiosyncrasy, there is again the hint of a pattern: the antonym pairs that diverge in their behavior tend to be dimensional adjectives (heavy/light, tall/short, big/small). Such pairs can be split into 'increasing-direction' adjective and a 'decreasing-direction' one (Seuren 1978); heavy is an increasing-direction adjective because heavier conveys more weight, light is decreasing-direction because lighter conveys less weight. Within these pairs, it is always the decreasing-direction one that prefers to be distributive - again suggesting that this behavior can be somehow tied to the similarities between these adjectives.

To explain these data, I propose an account using measurement theory (Section 3; Stevens 1946, Suppes and Zinnes 1962, Krantz et al. 1971, Roberts 1979, Schwarzschild 2002, Schwarzschild 2006, Sassoon 2007, Sassoon 2010, Lassiter 2011, Solt 2015, Lassiter 2017) - a system used to capture the properties of various systems of measurement, such as height, weight, temperature, likelihood, and so on. The idea is that for a gradable adjective $A$ to have a nondistributive understanding, the measurement along the scale encoded by $A$ of two things together $\mu(a \oplus b)$ must be able to exceed the measurement of each thing individually $(\mu(a)$ and $\mu(b))$. Then the contextual standard $\theta$ for what counts as $A$ can be set in such a way that $a \oplus b$ exceeds the standard $\theta$ for $A$ while $a$ and $b$ individually fall short of it - a nondistributive understanding, because $A$ is true of $a \oplus b$ together, but not of $a$ or $b$ alone. Depending on the behavior of the particular scale associated with the adjective, this ordering might or might not be possible, explaining which adjectives can or cannot be understood nondistributively.

2.Background on gradable adjectives and measurement theory. This paper focuses on gradable adjectives (Bartsch and Vennemann 1972, Seuren 1973, Cresswell 1976, Klein 1980,

\footnotetext{
${ }^{2}$ Scontras and Goodman describe adjectives such as light (decreasing-direction adjectives that are understood distributively) as 'stubbornly distributive', like tall - suggesting that light-type adjectives have an imaginable-butpragmatically-unavailable nondistributive understanding. But while it is clear how two boxes could be considered short individually and tall when stacked, it is not at all clear what it would mean for two boxes to be heavy individually and light together. Rather than grouping light with tall, I claim that light behaves like fragile in that it is difficult to even imagine how it could be understood nondistributively.
} 
Kennedy 1999, Kennedy 2007) - adjectives that can be degree-modified (very tall, somewhat heavy) and participate in comparative constructions (more beautiful, less full). Gradable adjectives are commonly analyzed (Cresswell 1976, von Stechow 1984, Rullmann 1995, Kennedy 1999 et seq.) to map an entity to its degree $d$ along the scale associated with the adjective, which (in the basic, non-comparative 'positive' form) is required to exceed some contextually determined threshold, represented as $\theta$. For example, (6) conveys that heavy applied to the box - the box's degree $d$ of weight - exceeds the contextual standard $\theta$ for what counts as heavy in the context.

$[[$ The box is heavy $]]=1$ iff heavy(the box $) \geq \theta$

Having introduced gradable adjectives, I turn to measurement theory (see Lassiter 2011 and Lassiter 2017: Chapter 2 for a comprehensive overview which inspires this discussion). Measurement theory is a system for representing measurements that does not take the (quantitative) natural numbers as basic, because natural numbers support relations - such as ratio comparisons - that do not carry over to certain qualitative orderings. For example, Room A may be warmer than Room B, but it does not strictly make sense to say that Room A is twice as warm as B. Even if Room A is 100 degrees Fahrenheit while Room B is 50 degrees Fahrenheit, this ratio is not preserved if we represent temperature in Celsius instead (then Room A is 38 degrees and Room B is 10 degrees). In this sense, temperature does not support the ratio comparisons that apply to natural numbers.

Instead of taking numbers as basic, measurement theory begins from the (qualitative) notion of relative ordering (which Sapir 1944 takes as psychologically basic): Does $a$ exceed $b$ with respect to the property being measured? Does $b$ exceed $a$ ? From these relative orderings, we construct a quantitative scale which preserves only as much structure from the natural numbers as is suitable for the property being measured.

Particularly important for analyzing distributivity, measurement theory can be used to represent how the measurement of an individual entity $a$ and of an individual entity $b$ relate to the measurement of a composite object, $a \bullet b$ (where $\bullet$ represents 'concatenation' - which, if $a$ and $b$ are not equal and do not overlap, is equivalent to the 'join' operation $\oplus$ of Link 1983; see Lassiter 2011: Chapter 2, building on Krifka 1989). Specifically, $\mu$ is a function mapping entities to the natural numbers in a way that preserves their relative ordering with respect to the property being measured; $\mu(a)$ is the quantitative measurement of an entity $a$ derived from its qualitative ranking relative to other entities. If $a$ outranks $b$ with respect to the property being measured, then $\mu(a)>\mu(b)$.

Using this system, it is possible to classify various types of scales (weight, temperature, and so on) based on the way $\mu(a \oplus b)$ relates to $\mu(a)$ and $\mu(b)$ - in other words, how the position of the composite entity $a \oplus b$ along the scale relates to the position of its constituent entities $a$ and $b$.

(7) Some types of scales adapted/abridged Lassiter (2011): 45

a. Additive: $\mu(a \oplus b)=\mu(a)+\mu(b)$ e.g. weight; weight $(a \oplus b)=$ weight $(a)+$ weight $(b)$

b. Intermediate: If $\mu(a)>\mu(b)$, then $\mu(a)>\mu(a \oplus b)>\mu(b)$ e.g. temperature; temp $(a \oplus b)$ falls between temp $(a)$ and temp $(b)$ 
c. Atomic Only: Ordering contains no concatenations; i.e. $\mu(a)>\mu(b)$ implies $a, b$ are atomic.

e.g. sick; $\operatorname{sick}(a \oplus b)$ is undefined because only individuals can be sick

For example, the scale of weight (7a) is classified as ADDITIVE, because the weight of two boxes $a$ and $b$ together is just the weight of the first box $a$ plus the weight of the other box $b$. In contrast, the scale of temperature (7b) is classified as INTERMEDIATE (at least, the thermometer temperature of non-chemically reactive substances - other construals of temperature are discussed further below): if one soup $a$ is 80 degrees Fahrenheit while another soup $b$ is 100 degrees Fahrenheit, then the composite $a \oplus b$ (the two soups mixed together) is certainly not 180 degrees Fahrenheit, but rather comes out to something like 90 degrees, falling in between the temperatures of the two constituent soups.

Lassiter (2011) also highlights scales that may be considered ATOM-ONLY, meaning that the scale is only sensible for non-composite, 'atomic' entities such as $a$ and $b$, not for composites such as $a \oplus b$. For example, perhaps it does not make sense to rate the sickness of two people together, given that only individuals can be sick. While other scales are classified by how $\mu(a \oplus b)$ relates to $\mu(a)$ and $\mu(b)$, here $\mu(a \oplus b)$ is undefined.

Before proceeding, it is worth noting that the superficially formal and precise concatenation operation '•’ (or equivalently, assuming no overlap, $\oplus$ ) actually requires some contextdependent, entity-specific decision-making about how the composite object $a \oplus b$ is to be assembled from its constituent parts $a$ and $b$. To measure the height of two boxes $a \oplus b$, do we measure them as a stack (in which case height is additive with respect to concatenation) or side by side and take the average (in which case height is intermediate)? To measure the temperature of two soups, do we mix them together or leave each one in its own container? If they are mixed together, could they react with one another chemically? What if the two elements being combined are of different types; what would it mean to concatenate a soup and a box? For current purposes, my approach is simply to articulate how I take the concatenation operation to work for the different types of composite entities that I discuss.

With that caveat, the next step is to use the classification laid out in (7) to derive the distributivity potential of adjectives from the way $\mu(a)$ and $\mu(b)$ relate to $\mu(a \oplus b)$ along the scale associated with the adjective.

3.The explanation. As observed in Section 1, heavy can be understood both distributively and nondistributively.

(8) Box A and Box B are heavy.

(can be understood both ways)

a. $\checkmark$ Distributive: Box A is heavy, Box B is heavy.

b. $\checkmark$ Nondistributive: Box A and Box B are jointly heavy but not individually so.

The analysis of gradable adjectives and measurement theory from Section 2 helps to explain why heavy can be understood in both ways. Imagine that Box A weighs 3lbs and Box B weighs 5lbs. For each box to individually qualify as heavy, each box's weight must exceed the contextual threshold $\theta$ for what counts as heavy in the context (the left side of Figure 1; the gray zone represents everything that is considered heavy, exceeding $\theta$ along the weight scale). 

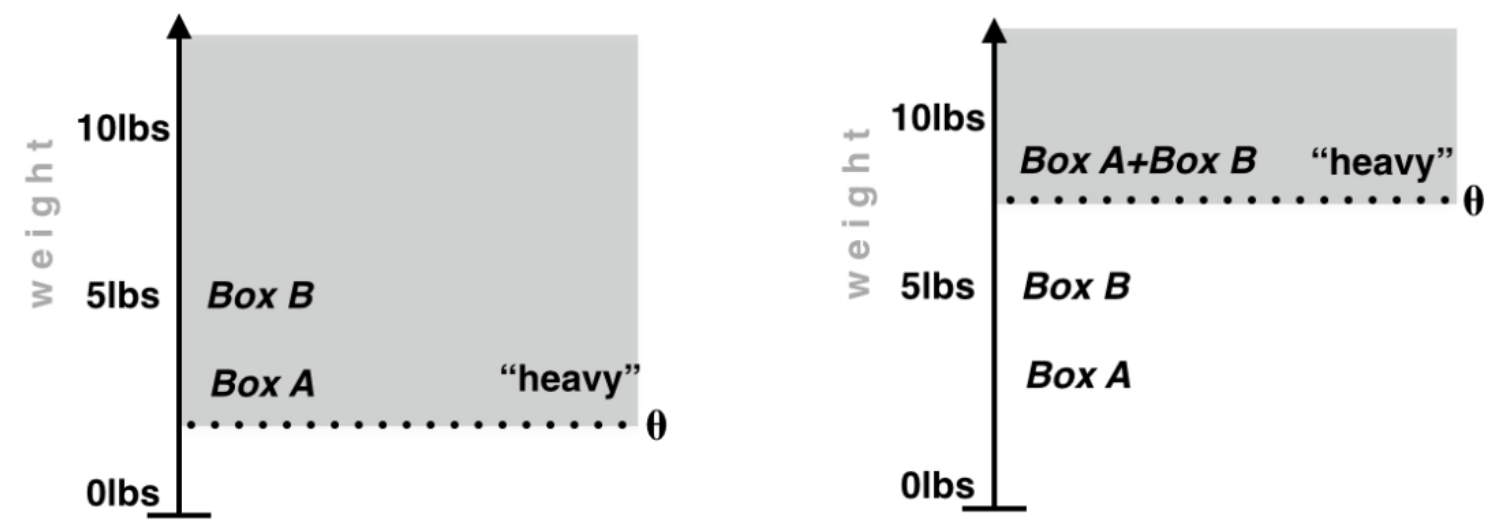

Figure 1. Distributive and nondistributive understandings of Box A and Box B are heavy.

For the two boxes to qualify as heavy jointly but not individually, the weight of $a \oplus b$ must exceed $\theta$ (so that the two boxes together are considered heavy), while the weight of each individual box falls short of $\theta$ (so that each individual box is not considered heavy). Weight is additive; the weight of $a \oplus b$ is the weight of a plus the weight of $\mathrm{b}$ (assuming that a weighs 3lbs and $\mathrm{b}$ weighs $5 \mathrm{lbs}$, then $a \oplus b$ weighs $8 \mathrm{lbs}$ ). Therefore, if the contextual standard $\theta$ for heavy is set at around 7lbs, then $a \oplus b$ exceeds it while $a$ and $b$ each fall short of it - a nondistributive understanding (the right side of Figure 1).

Of course, since $\theta$ depends on a comparison class (Klein 1980), it could theoretically be set at different levels when weighing a single box (compared to other individual boxes) versus when weighing a pair of boxes (compared to other pairs of boxes). But when heavy is understood nondistributively - when two boxes qualify as heavy while each individual box does not - it actually seems that the individual boxes $a$ and $b$ and the pair of boxes $a \oplus b$ are all compared to the same consistent standard (for example, 'what I can carry easily'). Otherwise, if Box A and Box B together are considered heavy relative to other pairs of boxes, then it seems likely that Box A and Box B would also each be considered heavy relative to other individual boxes. Such a variable setting of $\theta$ makes it much harder to imagine how heavy could be construed nondistributively.

Generalizing the discussion of heavy, I claim that any gradable adjective can only be understood nondistributively if $\mu(a \oplus b)$ exceeds $\mu(a)$ and $\mu(b)$ along the scale associated with the adjective (as in the right side of Figure 1). That way, $\theta$ can be set in such a way that $a \oplus b$ exceeds $\theta$, while $\mathrm{a}$ and $\mathrm{b}$ each fall short of it individually - nondistributive, because the adjective is true of $a \oplus b$ but not of $a$ or $b$. Depending on the behavior of a given adjective's associated scale with respect to the formation of composite objects, this arrangement may or may not be possible, explaining why different adjectives behave differently.

This analysis explains why other increasing-direction dimensional adjectives (tall, large, wide) behave like heavy in having an imaginable (more or less pragmatically available) 
nondistributive understanding. Height, size, and width - like weight - are additive, guaranteeing that $\mu(a \oplus b)$ exceeds $\mu(a)$ and $\mu(b)$, which is what is required for an imaginable nondistributive understanding. Other adjectives such as loud and expensive are not strictly additive (two 50decibel sounds do not create a 100-decibel sound in combination; two 50-dollar chairs might be discounted when purchased together), but the loudness or cost of two things together still generally exceeds that of each one individually, explaining why these adjectives behave like heavy in having an available nondistributive understanding.

As for why heavy is easily understood nondistributively while tall 'stubbornly' prefers to be distributive, I echo Scontras and Goodman's (2017) idea that the joint weight of boxes is more stable and thus easier to coordinate on pragmatically than joint height. What is new is that the proposed analysis also explains what unifies heavy and tall and separates them from fragilewhy both heavy and tall have an imaginable nondistributive understanding while fragile does not.

Unlike heavy, light is only understood distributively; it is very difficult to imagine how two individually heavy boxes could qualify as light when lifted together (Section 1). The proposed analysis helps to explain why. Imagine again that Box A is 3lbs while Box B is 5lbs, so that the two boxes together weigh $81 \mathrm{bs}$. Since the light scale measures weight in a decreasing directionwhere a higher position on the light scale entails less weight - the two boxes together $a \oplus b$ are guaranteed to be less light than each individual box along the scale (Figure 2). As a result, $\theta$ might be set so that $a, b$, and $a \oplus b$ all count as light, or so that only $a$ and/or $b$ count as light while $a \oplus b$ does not - but it cannot be set in such a way that $a \oplus b$ exceeds $\theta$ while $a$ and $b$ individually fall short of it, what would be required for a nondistributive understanding. This analysis therefore explains why decreasing-direction adjectives (light, short, quiet, cheap) are only understood distributively, whereas their increasing-direction antonyms can be understood in both ways.

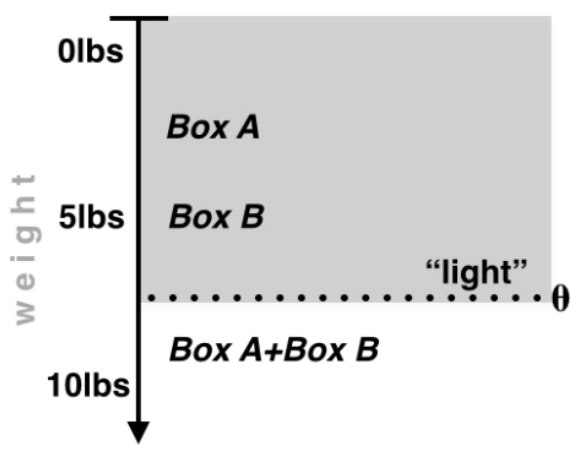

Figure 2. Two things together are no lighter than each one individually.

In contrast to weight, the scale of temperature (at least, the thermometer temperature of nonchemically reactive substances) is intermediate with respect to concatenation - the temperature of two soups mixed together falls in between the temperatures of the two constituent soups which explains why warm is only understood distributively in (9). 
(9) Soup A and Box B are warm.

(distributive)

a. VDistributive: Soup A is warm, Soup B is warm.

b. XNondistributive: Soup A and Soup B are jointly warm but not individually so.

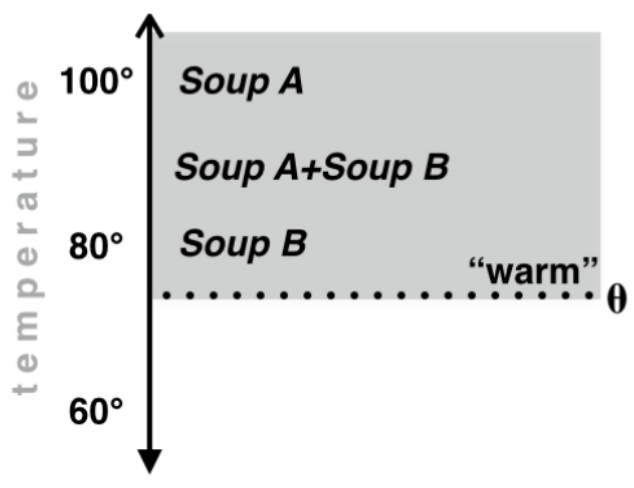

Figure 3. The temperature of two soups falls between the temperature of each one.

When $\mu(a \oplus b)$ falls in between $\mu(a)$ and $\mu(b)$ (intermediate; Figure 3), there is no way to set $\theta$ so that $a \oplus b$ exceeds it while $a$ and $b$ individually fall short of it - what would be required for a nondistributive understanding. All three entities $(a, b$, and $a \oplus b)$ might count as warm; or two of the three $(a \oplus b$ and either $a$ or $b$ ); or none of them (imagining different settings of $\theta$ in Figure 3); but if $a \oplus b$ qualifies as warm, then either $a$ or $b$ (or both) must also qualify. Therefore, warm cannot be understood in such a way that the two soups together qualify as warm while each soup individually does not. The same analysis also extends to other adjectives with scales that behave intermediately with respect to concatenation, such as clean and dirty.

So far, the discussion of temperature has been explicitly restricted to the thermometer temperature of non-reactive substances. Why only non-reactive substances? Because if two chemicals react with one another to produce heat, then the temperature of the two chemicals together may exceed the temperature of each one, yielding a nondistributive understanding. And why only thermometer temperature? Because temperature can be construed in different ways - as an objective numerical measurement; or as a subjective bodily experience, perhaps the tactile temperature of a specific object, or the ambient temperature of a room, or one's body temperature in relation to the comfortable range for humans (Koptjevskaja-Tamm and Rakhilina 2006, Koptjevskaja-Tamm 2011). Construed in these ways, temperature may not be intermediate with respect to concatenation: (10) may convey that a person only feels warm (comfortable in cold weather) when wearing a hat and a scarf together, not just one or the other (assuming that a person feels warmer with every piece of clothing they put on) - a nondistributive understanding of warm.

(10) The hat and the scarf are warm.

(can be understood both ways)

a. VDistributive: The hat is warm, the scarf is warm.

b. $\checkmark$ Nondistributive: The hat and the scarf are jointly warm but not individually so. 
In other words, depending on the nature of concatenation (whether it involves a chemical reaction or not) and on the construal of temperature (thermometer vs. subjective experience), temperature behaves differently with respect to concatenation in different contexts. In turn, the way temperature behaves with respect to concatenation influences the distributivity potential of temperature adjectives such as warm. When temperature is intermediate with respect to concatenation, warm is only understood distributively. When it is additive, warm can be understood nondistributively, because $a \oplus b$ can be considered warm while $a$ and $b$ individually do not qualify. In other words, the distributivity potential of an adjective is not a lexical fact about the specific adjective, nor is it fully predicted from the property (e.g. temperature) measured by it. Consistent with the proposed analysis, what is most important is the way $a \oplus b$ relates to $a$ and $b$ along the scale associated with the adjective.

As previewed above (Section 1), the subjective adjectives beautiful and ugly can be understood both distributively and nondistributively.

(11) Box A and Box B are beautiful.

(can be understood both ways)

a. VDistributive: Box A is beautiful, Box B is beautiful.

b. VNondistributive: Box A and Box B are jointly beautiful but not individually so.

Again, these facts follow from the behavior of these scales with respect to concatenation. Beauty and ugliness are subjective (Lasersohn 2005), tied to some party's taste and judgment. Such scales behave irregularly with respect to concatenation: two boxes might be individually beautiful, even more beautiful together; individually beautiful, but ugly when arranged together; individually ugly, but beautiful together; and so on - anything is possible. As a result, two boxes might each be beautiful or ugly (distributive), or might only become so when they complement one another (nondistributive). The same analysis also extends to other subjective adjectives, such as delicious and disgusting, which can similarly be construed both distributively and nondistributively.

So far, most of the adjectives discussed in this paper have described properties (height, weight, temperature, beauty) that can be instantiated by pluralities as well as individuals. But there are also properties that can only really be instantiated by individuals (sick, alive, grayhaired, worried, religious, American ...), given that individuals have their own bodies/hair, beliefs, and nationalities. Such adjectives are only understood distributively:

(12) Alice and Bob are \{sick, religious, American...

(distributive)

a. VDistributive: Each of them is \{sick, religious, American... $\}$.

b. XNondistributive: Jointly $\{$ sick, religious, American... $\}$ but not individually so.

Lassiter (2011): Chapter 2 suggests that the sickness of two individuals $a \oplus b$ together is undefined, given that only individuals can be sick (Lassiter's 'atom-only' scales). The ordering required for a nondistributive understanding - where $\mu(a \oplus b)$ exceeds $\mu(a)$ and $\mu(a \oplus b)$ - is not possible if $\mu(a \oplus b)$ is undefined. Therefore, the proposed measurement-theoretic account explains why these sick-type adjectives are only understood distributively.

For some adjectives (fragile, full, empty), it is difficult to say whether they should be considered 'atom-only', like sick; or intermediate with respect to concatenation, like warm. For example: recall that, applied to two boxes, fragile is understood distributively (Section 1). If 
fragile is considered atom-only (on the grounds that it describes physical material, which individual boxes possess individually), then it is understood distributively for the same reason that sick is. If it is considered intermediate with respect to concatenation (on the grounds that the fragility of two boxes together falls in between the fragility of each one), then it behaves like warm. The reasoning is different, but the same prediction is made either way: fragile is understood distributively.

Stepping back, the proposed analysis provides a predictive, explanatory answer to the question of 'which adjectives are understood in which ways, and why?'. As sketched in Table 2, fragile-type adjectives (above the bolded line) are understood distributively because $a \oplus b$ cannot surpass $a$ and $b$ along their associated scales. The heavy and tall-type adjectives (below the bolded line) have an imaginable (more or less pragmatically available) nondistributive understanding because $a \oplus b$ can exceed $a$ and $b$ along the scale, so that the contextual standard $\theta$ can be set in such a way that $a \oplus b$ surpasses it while $a$ and $b$ each individually fall short.

\begin{tabular}{|c|c|}
\hline Distributive & Box $A$ and Box B are fragile (light, short, full, empty, old, new). \\
\hline & $\checkmark$ Dist.: each fragile $\quad$ XNondist.: jointly fragile \\
\hline & because $a \oplus b$ can't exceed $a, b$ on fragile scale \\
\hline Both ways & Box $A$ and Box B are heavy (expensive, beautiful, ugly). \\
\hline & VDist.: each heavy $\quad \checkmark$ Nondist.: jointly heavy \\
\hline & $\begin{array}{l}\text { because } a \oplus b \text { can exceed } a, b \text { on heavy scale } \\
\text { (pragmatically available because joint weight is stable) }\end{array}$ \\
\hline 'Stubbornly & Box $A$ and Box B are tall (big, large, long, wide). \\
\hline distributive' & $\checkmark$ Dist.: each tall $\quad$ (??) Nondist.: jointly tall \\
\hline & because $a \oplus b$ can exceed $a, b$ on tall scale \\
\hline & (pragmatically unavailable because joint height is unstable) \\
\hline
\end{tabular}

Table 2: Distributive and nondistributive understandings available to different adjectives, and how these understandings are explained on the proposed analysis.

4.Conclusion. This paper has focused on the distributivity potential of adjectives, but the same phenomenon is also observed among Verb Phrases (VPs; which in fact have received relatively more attention in the distributivity literature). For example, the verb smile behaves like the adjective fragile in that it is only understood distributively (13), whereas the VP lift the box behaves like heavy in that it can be understood both distributively and nondistributively (14).

(13) Alice and Bob smiled.

(distributive)

a. VDistributive: Alice smiled, Bob smiled.

b. XNondistributive: Alice and Bob smiled jointly but not individually.

(14) Alice and Bob lifted the box.

(can be understood both ways)

a. VDistributive: Alice lifted the box, Bob lifted the box.

b. $\checkmark$ Nondistributive: Alice and Bob lifted the box jointly but not individually.

Just as the distributivity potential of an adjective depends on what we know about the nature of the property it describes, the distributivity potential of a VP depends on what we know about 
the event (Scha 1981, Roberts 1987, Dowty 1987, Winter 2000). Smiling involves the face; people have their own faces, so they can only smile individually; boxes can be lifted individually or multi-laterally. Against this background, the current study of distributivity among adjectives fits into a larger investigation of the distributivity potential of various types of predicates from different syntactic categories (Glass to appear). The specific explanations take distinct forms because the scalar notions described by gradable adjectives differ from the events described by VPs; but in both cases, the goal is to make the idea that a predicate's distributivity potential 'depends on world knowledge' predictive by pinpointing the facets of world knowledge that matter.

\section{References}

Bartsch, Renate, and Theo Vennemann. 1972. The grammar of relative adjectives and comparison. Linguistische Berichte (Linguistic Reports) 20. 19-32.

Champollion, Lucas. 2010. Parts of a whole: Distributivity as a bridge between aspect and measurement. Philadelphia, PA: University of Pennsylvania dissertation.

Champollion, Lucas. 2017. Parts of a whole: distributivity as a bridge between aspect and measurement. Oxford Studies in Theoretical Linguistics. Oxford, UK: Oxford University Press. https://doi.org/10.1093/oso/9780198755128.001.0001.

Clark, Eve V. 1972. On the child's acquisition of antonyms in two semantic fields. Journal of Verbal Learning and Verbal Behavior, 11(6). 750-758. https://doi.org/10.1016/ S0022-5371(72)80009-4.

Cresswell, Maxwell John. 1976. The semantics of degree. In Barbara Partee (ed), Montague Grammar. 261-292. New York: Academic Press. https://doi.org/10.1016/ B978-0-12-545850-4.50015-7.

Cruse, D. Alan. 1976. Three classes of antonym in English. Lingua, 38(3-4), 281-292. https:// doi.org/10.1016/0024-3841(76)90015-2.

de Vries, Hanna. 2015. Shifting sets, hidden atoms: The semantics of distributivity, plurality and animacy. Utrecht, Netherlands: University of Utrecht dissertation.

Dowty, David R. 1987. A note on collective predicates, distributive predicates, and all. In Fred Marshall (ed), Proceedings of the Third Eastern States Conference on Linguistics (ESCOL). 95-115. Columbus, Ohio: The Ohio State University.

Glass, Lelia. To appear. Exploring the relation between argument structure and distributivity. In Julia Nee, Margaret Cychosz, Dmetri Hayes, Tyler Lau, and Emily Remirez (eds), Proceedings of the 43rd Annual Meeting of the Berkeley Linguistics Society. eScholarship.

Kennedy, Christopher. 1999. Projecting the adjective: The syntax and semantics of gradability and comparison. New York, NY: Garland.

Kennedy, Christopher. 2007. Vagueness and grammar: The semantics of relative and absolute gradable adjectives. Linguistics and Philosophy 30(1). 1-45. https://doi.org/10.1007/ s10988-006-9008-0.

Klein, Ewan. 1980. A semantics for positive and comparative adjectives. Linguistics and Philosophy 1(4). 1-45. https://doi.org/10.1007/BF00351812. 
Koptjevskaja-Tamm, Maria. 2011. 'It's boiling hot': On the structure of the linguistic temperature domain across languages. In Sarah Dessì Schmid, Ulrich Detges, Paul Gévaudan, Wiltrud Mihatsch, and Richard Waltereit (eds), Rahmen des Sprechens: Beiträge zur Valenztheorie, Varietätenlinguistik, Kognitiven und Historischen Semantik (Frame of speech: Contributions to Valence Theory, Variationist Linguistics, Cognitive and Historical Semantics). 393-410. Tübingen: Narr.

Koptjevskaja-Tamm, Maria, and Ekaterina V. Rakhilina. 2006. 'Some like it hot': On semantics of temperature adjectives in Russian and Swedish. In Giannoula Giannoulopoulou and Torsten Leuschner (eds), STUF (Sprachtypologie und Universalienforschung / Language Typology and Universals), a special issue on the Lexicon in a Typological and Contrastive Perspective. 253-269. https://doi.org/10.1524/stuf.2006.59.3.253.

Krantz, David H., R. Duncan Luce, Patrick Suppes, and Amos Tversky. 1971. Foundations of Measurement. Academic Press.

Krifka, Manfred. 1989. Nominal reference, temporal constitution and quantification in event semantics. In Renate Bartsch, Johan van Benthem, and Peter van Emde Boas (eds), Semantics and contextual expression. 75-115. Stanford, CA: CSLI Publications.

Landman, Fred. 1989. Groups, I. Linguistics and Philosophy 12(5). 559-605. https:/doi.org/ 10.1007/BF00627774.

Landman, Fred. 2000. Events and Plurality: The Jerusalem Lectures. Dordrecht: Kluwer. https:// doi.org/10.1007/978-94-011-4359-2.

Lasersohn, Peter. 1995. Plurality, Conjunction and Events. Dordrecht, The Netherlands: Kluwer. https://doi.org/10.1007/978-94-015-8581-1.

Lasersohn, Peter. 2005. Context dependence, disagreement, and predicates of personal taste. Linguistics and philosophy, 28(6), 643-686. https://doi.org/10.1007/s10988-005-0596-X.

Lassiter, Daniel. 2011. Measurement and Modality. Ph.D. thesis, New York University.

Lassiter, Daniel. 2017. Graded Modality: Qualitative and Quantitative Perspectives. Oxford, U.K.: Oxford University Press. https://doi.org/10.1093/oso/9780198701347.001.0001.

Lehrer, Adrienne, and Keith Lehrer. 1982. Antonymy. Linguistics and Philosophy 5(4). 483501. https://doi.org/10.1007/bf00355584.

Link, Godehard. 1983. The logical analysis of plurals and mass terms: A lattice-theoretical approach. In Rainer Baeuerle, Christoph Schwarze, and Arnim von Stechow (eds), Meaning, Use and Interpretation of Language. 302-323. Berlin: Walter de Gruyter. https://doi.org/ $10.1515 / 9783110852820$.

Maldonado, Mora, Emmanual Chemla, and Benjamin Spector. 2018. Priming as a window to the collective/distributive ambiguity of adjectival predicates. Abstract/poster presented at Linguistic Evidence, Tübingen.

Muehleisen, Victoria Lynn. 1997. Antonymy and semantic range in english. Evanston, IL: Northwestern University dissertation.

Roberts, Craige. 1987. Modal Subordination, Anaphora and Distributivity. Amherst, MA: University of Massachusetts dissertation.

Roberts, Fred S. 1979. Measurement theory with applications to decisionmaking, utility, and the social sciences. Addison Wesley Publishing Company. 
Rullmann, Hotze. 1995. Maximality in the semantics of wh-constructions. Amherst, MA: University of Massachusetts dissertation.

Sapir, Edward. 1944. Grading: A study in semantics. Philosophy of Science 11. 93-116. https:// doi.org/10.1086/286828.

Sassoon, Galit Weidman. 2007. Vagueness, gradability and typicality: A comprehensive semantic analysis. Tel Aviv, Israel: Tel Aviv University dissertation.

Sassoon, Galit Weidman. 2010. Measurement theory in linguistics. Synthese, 174(1), 151-180. https://doi.org/10.1007/s11229-009-9687-5.

Scha, Remko. 1981. Distributive, collective and cumulative quantification. In Jeroen Groenendijk, Theo Janssen, and Martin Stockhof (eds), Formal methods in the study of language. 483-512. Amsterdam: Mathematical Center Tracts.

Schwarzschild, Roger. 1996. Pluralities. Dordrecht; Boston: Kluwer Academic. https://doi.org/ 10.1007/978-94-017-2704-4.

Schwarzschild, Roger. 2002. The grammar of measurement. In Brendan Jackson (ed), Semantics and Linguistic Theory (SALT) 12. 225-245. Ithaca, NY: Cornell University. https://doi.org/ 10.3765/salt.v12i0.2870.

Schwarzschild, Roger. 2006. The role of dimensions in the syntax of noun phrases. Syntax 9(1). 67-110. https://doi.org/10.1111/j.1467-9612.2006.00083.x.

Schwarzschild, Roger. 2011. Stubborn distributivity, multiparticipant nouns and the count/mass distinction. In Brian Smith, Suzi Lima, and Kevin Mullin (eds), Proceedings of the North East Linguistic Society (NELS) 39. 661-678. Ithaca, NY: Cornell University.

Scontras, Gregory, and Noah Goodman. 2017. Resolving uncertainty in plural predication. Cognition 168. 294-311. https://doi.org/10.1016/j.cognition.2017.07.002.

Seuren, Pieter A. M. 1978. The structure and selection of positive and negative gradable adjectives. In Donka Farkas, Wesley M. Jacobsen, and Karol W. Todrys (eds), Papers from the Parasession on the Lexicon, Proceedings of the Chicago Linguistic Society (CLS) 14. 336-346. Chicago, IL: University of Chicago.

Seuren, Pieter A.M. 1973. The comparative. In Ferenc Keifer, Ferenc and Nicolas Ruwet (eds), Generative grammar in Europe. 528-563. Dordrecht: Reidel. https://doi.org/ 10.1007/978-94-010-2503-4_22.

Solt, Stephanie. 2015. Measurement scales in natural language. Language and Linguistics Compass 9(1). 14-32. https://doi.org/10.1111/lnc3.12101.

Stevens, Stanley Smith. 1946. On the theory of scales of measurement. Science 103. 688-680. https://doi.org/10.1126/science.103.2684.677.

Suppes, Patrick, and Joseph L. Zinnes. 1962. Basic measurement theory. Palo Alto, CA: Institute for Mathematical Studies in the Social Sciences, Stanford University.

Syrett, Kristen. 2015. Mapping properties to individuals in language acquisition. Proceedings of the Annual Boston University Conference on Language Development BUCLD (39). 398-410. Boston, MA: Cascadilla Press. https://doi.org/doi:10.7282/T3KD20XC.

von Stechow, Arnim. 1984. Comparing semantic theories of comparison. Journal of Semantics 3. 1-77. https://doi.org/10.1093/jos/3.1-2.1.

Winter, Yoad. 2000. Distributivity and dependency. Natural Language Semantics, 8(1). 27-69. https://doi.org/10.1023/A:1008313715103. 